\title{
«CONDUIRE À CELA QUI EST REFUSÉ AU MOT» WALTER BENJAMIN OU LA CRITIQUE DE L'IDÉOLOGIE DE LA COMMUNICATION
}

\author{
Marc Berdet ${ }^{l}$
}

Resumé: Walter Benjamin est de plus en plus présent dans les départements de communication aujourd'hui, et parfois même utilisé dans le discours des communicants eux-mêmes. Pourtant, quelque chose en lui résiste à cette instrumentalisation: c'est que, dans son œuvre, le langage (politique comme esthétique) ne sert pas à communiquer un sens prédéfini, mais à exprimer cela même qui est refusé au mot. Le philosophe allemand va jusqu'à liquider la posture révolutionnaire de l'artiste encore pris dans l'idéologie de la communication: il ne s'agit ni de remplacer le contenu, ni de rénover la forme, mais, objectif apparemment modeste mais radicalement plus ambitieux, de changer la technique.

Mots-Clés: Walter Benjamin. Communication. Art Langage. Révolution.

\section{INTRODUCTION: AU CAFÉ TIERGARTEN}

Berlin, café Tiergarten, un jour d'automne 1913. Walter Benjamin, 21 ans à peine, et Gershom Scholem, presque 16 ans, se croisent pour la première fois. Tous deux participent à une rencontre politique entre un cercle de jeunes sionistes et de jeunes adeptes d'une pédagogie alternative. ${ }^{2}$ Porte-parole de ces derniers, Benjamin intervient pour, sans rejeter frontalement le sionisme d'avant-guerre auquel adhère le cénacle de Scholem, "l'évacuer" (SCHOLEM, 1981, p. 12) au fil d'un discours pénétrant. Scholem ne peut pas se rappeler de la teneur de son argument, mais reste marqué par la manière de parler de cet étrange orateur:

Il discourait avec une grande intensité, et en s'exprimant de façon parfaite, sans regarder l'auditoire; en fait, ses yeux fixaient en permanence un coin

\footnotetext{
${ }^{1}$ Marc Berdet, Departamento de sociologia - Faculdade de Filosofia, Letras e Ciências Humanas Universidade de São Paulo (processo no 2015/04514-4, Fundação de Amparo à Pesquisa do Estado de São Paulo). Auteur en français de Fantasmagories du capital. L'invention de la ville-marchandise (Paris: La Découverte, 2013); Walter Benjamin. La passion dialectique (Paris, Armand Colin, 2014); Le chiffonnier de Paris. Walter Benjamin et la fantasmagorie (Paris: Vrin, 2015); et, en français et en allemand, avec Thomas Ebke, de Matérialisme anthropologique et matérialisme de la rencontre. Arpenter notre présent avec Walter Benjamin et Louis Althusser (Berlin: Xenomoi, 2014). E-mail: marcberdet@ gmx.com

${ }^{2}$ Il s'agit de la branche du Mouvement de la jeunesse regroupée autour du pédagogue Gustav Wyneken. Pour cet épisode, le rapport de Benjamin au sionisme et son activisme dans le Jugendbewegung, voir Eiland et Jennings (2014, p. 32-74) et Berdet (2014, p. 25-41).
}

http://dx.doi.org/10.1590/S0101-31732017000300004 
du plafond de la salle, comme s'il s'adressait à un auditeur bizarrement placé en cet endroit. Je ne me rappelle plus non plus ce que lui répliquèrent ses contradicteurs sionistes. (SCHOLEM, 1981, p. 12).

Cette anecdote contient en germe le geste si particulier des engagements futurs du philosophe juif et marxiste hétérodoxe Walter Benjamin. Quel est donc cet intellectuel étrange qui, plutôt que de s'adresser à ses camarades pour les convaincre, préfère parler à un coin du plafond ? Ne connaît-il pas les bases de la communication, qui veut que l'on regarde ceux que l'on souhaite convaincre ? Le face-à-face de la confrontation virile d'opinions lui semblet-il stérile ? "Convaincre est infécond" (BENJAMIN, 2013c, p. 143), lit-on comme en réponse dans un fragment publié en 1928 intitulé Pour hommes. C'est, en effet, une constante du travail de Benjamin que de chercher à ébranler son lecteur autrement que par la présentation didactique d'arguments clairement enchaînés. La préface à sa thèse d'habilitation de 1925, pour prendre l'exemple le plus évident, n'a rien d'une introduction au sens pédagogique du terme (BENJAMIN, 2000b, p. 23-56). Elle se veut, de l'avis du candidat luimême, une "haie d'épines" (BENJAMIN, 1979, p. 340) destinée à décourager le lecteur. Curieux début concernant une habilitation à diriger les recherches nécessaire pour faire une carrière universitaire en Allemagne. Elle aurait dû au contraire faciliter la tâche de ses futurs rapporteurs et leur communiquer les données essentielles de la thèse du candidat, de sa position et de son apport. Mais non. Benjamin y présente une théorie mystique du langage "maquillée" en une non moins complexe "théorie des idées" BENJAMIN, (1979, p. 340). Il ne se soucie pas de convaincre par la discussion rationnelle, mais de faire sentir, à travers le texte, l'éclair de la vérité (ici quant à l'origine du baroque), comme le mentionne une note préparatoire du Livre des passages consignée une dizaine d'années plus tard: "Dans les domaines qui nous occupent, il n'y a de connaissance que fulgurante. Le texte est le tonnerre qui fait entendre son grondement longtemps après." (BENJAMIN,1989, p. 473)

Peut-être ne serait-il pas géré de voir l'explication avec le sionisme comme le texte de Scholem qui résonne longtemps après sa rencontre avec Benjamin. Mais il importe surtout ici que Scholem se souvienne de l'éclair (l'intensité du discours, la perfection de l'expression) plus que du texte (le contenu de la discussion elle-même). Éclair d'un "message" qui passait non pas entre Benjamin et ses camarades mais, au travers de ses camarades, entre Benjamin et un coin du plafond où un mystérieux auditeur semblait s'être 
logé. Qu'est-ce que le jeune philosophe voulait-il alors transmettre ? À cette époque, il considérait la classe étudiante comme la " grande transformatrice de l'histoire" (BENJAMIN, 2000a, v. I, p. 135); plus tard, ce sera au prolétariat de jouer ce rôle dans sa pensée politique. Au fond, sa manière d'intervenir en 1913 caractérise un style qui, de l'engagement juvénile dans le Mouvement de la jeunesse des années 1910 à la solidarité avec l'avant-garde révolutionnaire des années 1930, ne cesse jamais d'être politique.

Nous résumerons la position de Benjamin de la manière suivante. Même si cela ne renvoie pas à ce que l'on entend habituellement par ce qualificatif, la nécessité constante d'intervenir par un style qui se veut politique persiste pardelà la rupture entre une jeunesse "idéaliste" et une maturité "matérialiste". 3 (1). Ce mode politique d'intervention sur l'esthétique s'affine et devient plus explicite au fil de la lutte antifasciste: d'une part, le philosophe rejette l'attitude qui se satisfait de la révolution des formes, et qui aboutit, dans le contexte des années 1930, à une rénovation superficielle (2); d'autre part, il écarte la position qui se contente de la révolution du contenu, et qui revient à remplacer le sujet de l'œuvre d'art sans que rien ne soit changé par ailleurs (3). Il propose d'intervenir plus radicalement dans le tissu des rapports de production - qui est aussi celui du langage - par une transformation au niveau technique, bouleversant à la fois forme et contenu, et transférant au collectif producteur la capacité individuelle de création (4). Il rejoint alors une conception de la technique et du langage qui présidait à ses premiers écrits, et dont la critique du paradigme bourgeois de la communication reste encore valable aujourd'hui.

\section{Un STYLE POLITIQUE}

Un style politique, donc, mais indirect. Au café Tiergarten, le jeune Benjamin voulait non pas communiquer une conviction, mais exprimer

\footnotetext{
${ }^{3}$ Benjamin lui-même utilise ces termes et souligne la rupture. Il note, en faisant implicitement référence à la dialectique matérialiste qui opposait Marx à Hegel et sa dialectique idéaliste, que son œuvre d'avant 1925 était "déjà dialectique", mais "certainement pas matérialiste" (BENJAMIN, 1980, p. 43). Origine du drame baroque allemand se présente en effet, nous l'avons vu, comme une "théorie des idées" (BENJAMIN, 1979, p. 340), et plus exactement comme une quête dialectique des "idées" du baroque sous le patronage de Hegel, Leibniz et Platon (BENJAMIN, 2000b, p. 43-44). Et c'est en 1924, alors qu'il finit de rédiger cet ouvrage, que Benjamin voit des "clignotants communistes" allumés sur sa route et qu'il change "[...] au contact d'une théorie bolchevique radicale" (benjamin 1980, p. 136) incarnée non seulement par Asja Lacis, partisane d'un théâtre prolétarien dont il tombe amoureux, que par Ernst Bloch et Georg Lukács, dont il découvre les livres sur l'utopie et la conscience de classe. Il rencontre peu après Bertolt Brecht et les membres de l'École de Francfort. (BERDET, 2014, p. 13-24; BERDET, 2015).
} 
une idée pour elle-même, au-delà de ses potentiels destinataires. Une telle intention rappelle une phrase dérangeante de son essai sur la traduction ${ }^{4}$ : "Car aucun poème ne s'adresse au lecteur, aucun tableau au spectateur, aucune symphonie à l'auditoire.” (BENJAMIN, 2000a, v. I, p. 244). Pour Benjamin, le poète, le peintre ou le musicien ne cherche pas à atteindre son destinataire en se servant de mots, de couleurs ou de sons comme de véhicules. Il cherche plutôt à exprimer, au sein des mots, des couleurs et des sons eux-mêmes, quelque chose de l'ordre de l'indicible, à donner aux mots, aux couleurs et aux sons quelque chose qui leur était refusé jusque-là. De manière similaire, le philosophe écrit dans ses notes préparatoires sur Paris: “[...] je n'ai rien à dire, seulement à montrer." (BENJAMIN, 1989, p. 476). De même que Origine du drame baroque allemand consistait dans le montage de près de 600 citations (BENJAMIN, 1979, p. 311), le Livre des passages devait en effet arracher des fragments à près de 800 sources afin d'en effectuer un montage littéraire. Ce geste par lequel l'écrivain - qu'il se fasse critique d'art (sur le baroque) ou historien (sur Paris) - intègre une technique extérieure (le montage cinématographique) témoigne de sa manière d'intervenir dans les formes d'écriture. L'intégration d'un élément étranger transforme l'écriture elle-même, dont le sens n'est plus délivré par le commentaire en surplomb (réintroduit en voix off au cinéma) mais par l'entrechoc des séquences (alors nouveau avec le cinéma encore muet).

Si aucun poème ne s'adresse au lecteur, il semble conséquent qu'aucun discours ne s'adresse au militant. Pas étonnant si Benjamin ne s'adresse pas à ses camarades, mais à un coin du plafond. Peut-être que le mystérieux auditeur qui, comme Scholem le devine, s'y loge, n'est rien moins que l'ordre du sensible lui-même qu'il s'agit de bouleverser. Cette tâche, à laquelle on reconnaît aisément le poète au travail, est paradoxalement politique pour Benjamin, et cela avant même son tournant marxiste de 1924. En témoigne sa lettre à Buber de 1916, par laquelle il signe son refus de participer à la revue Le juif qui s'engageait alors dans le sionisme. Peut-être qu'il y reprend sa critique, en effet étonnante, oubliée par Scholem (1979, p. 117):

Ma notion d'un style et d'une écriture objective, par là même hautement politique, est celle-ci: conduire à cela qui est refusé au mot; là où cette sphère de défaillance du langage éclate avec une puissance qu'aucun mot ne peut dire, là seulement peut jaillir entre le mot et l'acte dynamisant l'étincelle magique, qui est l'unité de l'un et de l'un et l'autre également effectif.

\footnotetext{
${ }^{4}$ Pour un commentaire éclairant de ce texte, cf. Berman (2008, p. 47 et suivantes).
} 
L'écriture politique ne consiste pas, pour Benjamin, à développer des arguments en faveur d'un projet étatique (ici le sionisme), c'est-à-dire à se servir des mots comme moyens en vue de fins qui leurs sont étrangères. Elle revient plutôt à activer une dynamique au sein du langage ("acte dynamisant") pour donner aux mots un sens qu'ils n'avaient pas jusqu'alors, qui leur était même refusé" par leur usage habituel, c'est-à-dire par les conventions.

On sera peut-être étonné d'apprendre ici que c'est la même nécessité politique qui pousse Benjamin à écrire “ L'œuvre d'art à l'époque de sa reproductibilité technique." ${ }^{5}$ En cela, n’en déplaise à des lecteurs déçus comme Hannah Arendt (1974) ou Gershom Scholem (1981 et, avec Benjamin (2011), notamment la postface de Stéphane Moses (p. 308-310), ce texte de maturité ne témoigne pas du sacrifice d'un philosophe du langage sur l'autel du militantisme marxiste de l'époque. Il s'agit au contraire d'une reprise d'un motif ancien: l'intervention active sur les formes du langage. Cette reprise se fait certes dans un contexte plus immédiatement politique, celui de la politisation communiste de l'esthétique (Bertolt Brecht comme allié) en lutte contre l'esthétisation fasciste de la politique (Filippo Tommaso Marinetti comme adversaire $)^{6}$. Mais c'est en tant que reprise, précisément, que cette intervention fait bouger les lignes rigidifiées des querelles militantes autour de la politisation de l'art. Ce "bougé" (cette voie transversale) a traversé le siècle, et sa justesse nous atteint encore aujourd'hui avec force, remettant sur la table les illusions quant à la tentative de créer une "prise de conscience" chez le public pour amorcer sinon un processus révolutionnaire, du moins un début de résistance à l'esthétisation (fasciste) du politique comme à l'esthétisation (capitaliste) de l'économie politique, bref à l'exploitation économique et aux oppressions politiques auxquelles elle peut conduire.

L'exposé le plus clair et - une fois n'est pas coutume - le plus pédagogique de cette position se trouve dans un autre texte d'intervention lui aussi honni par Arendt et Scholem (non sans raison puisque l'argument de jeunesse s'y trouve complètement sécularisé). Il s’agissait de notes destinées à être lues le 27 avril 1934 à l'Institut pour l'étude du fascisme de Paris en présence d'autres intellectuels antifascistes. Dans ce texte intitulé "L'auteur

\footnotetext{
${ }^{5}$ 2013a pour les cinq versions; 2000a, v. III, p. 67-113 et 269-316 pour les deuxième et cinquième versions; 1991, p. 140-171 pour la quatrième version en français.

${ }^{6}$ La première version est, politiquement, la moins ambiguë: "Voilà l'esthétisation de la politique que pratique le fascisme. Le communisme y répond par la politisation de l'art." (BENJAMIN, 2003, v. III, p. 113). Sur les différentes versions et la censure d'Horkheimer, voir Berdet (2014, p. 205-211) et Tackels, (2001).
} 
comme producteur." Benjamin (2003, p. 122-144), entreprend d'éclairer les intellectuels sur leur condition de producteurs, déterminante pour leurs propres travaux sans qu'ils en aient forcément conscience, pris qu'ils sont dans l'éternel débat de la forme et du contenu et la discussion plus récente de la position de leurs œuvres vis-à-vis des rapports de production (en deux mots: critique ou apologie ?). Le texte s'attèle, aux fins d'ouvrir une troisième voie, à départager clairement les deux visions militantes qui s'affrontent lorsqu'il s'agit de conférer à l'art une fonction politique de résistance à l'oppression et aux conformismes de toutes sortes. Ces deux visions renvoient, y compris dans leurs versions plus sophistiquées, à l'opposition de la forme et du contenu que Benjamin (2000a, v. III, p. 69) tient, dans "L'œuvre d'art à l'époque de sa reproductibilité technique", pour stérile et même dangereuse car susceptible d'être instrumentalisée par les fascismes. Pourtant, lorsqu'ils cherchent à politiser l'art, les artistes antifascistes n'en sortent pas toujours: ils se limitent trop souvent, dans leur volonté d'ébranler les rapports de production (l'ordre économique et politique) et de dépasser la simple révolution avant-gardiste des formes, à la simple révolution du contenu, c'est-à-dire à simplement placer l'ouvrier et les nouvelles conditions misérables de production au centre de l'œuvre. Parce que ses auditeurs connaissent l'argument, Benjamin ne s'attarde pas, dans "L'auteur comme producteur", sur sa critique de la position avantgardiste. Il est pourtant nécessaire de la déployer avant de pouvoir dépasser l'autre écueil, celui de l'art prolétarien.

\section{RÉNOVER LA FORME}

C'est dans les notes préparatoires au Livre des passages qu'on saisit combien la révolution avant-gardiste des formes se réduit bien souvent à une rénovation de façade complice d'une ré-auratisation réactionnaire de l'œuvre d'art telle qu'exposée dans "L'œuvre d'art à l'époque de sa reproductibilité technique". Benjamin y écarte l'idée qu'il suffirait de transformer la forme, typique des " conceptions que propage l'avant-garde bourgeoise. " (BENJAMIN, 1989, p. 413). Il lit dans Europe, revue littéraire proche des communistes qui circulait parmi les intellectuels antifascistes présents au congrès, l'argument suivant qu'il s'empresse de recopier:

La confusion du mot révolution qui, pour un léniniste, signifie la conquête du pouvoir par le prolétariat et qui signifie, par ailleurs, le bouleversement des valeurs spirituelles admises, les surréalistes la soulignent assez par leur désir de montrer Picasso comme un révolutionnaire... Picasso les déçoit... 
un peintre n'est pas plus révolutionnaire pour avoir «révolutionné» la peinture, qu'un couturier comme Poiret pour avoir «révolutionné» la mode ou qu'un médecin pour avoir «révolutionné» la médecine. (BENJAMIN, 1989, p. 413). ${ }^{7}$

Le philosophe juge ce diagnostic "parfaitement exact" (BENJAMIN, 1989, p. 413). Ce n'est pas, donc, parce qu'un artiste est révolutionnaire en art qu'il l'est forcément dans la société; ce n'est pas parce que Picasso révolutionne les formes de la peinture qu'il est d'une quelconque utilité pour le mouvement social. Dans "L'œuvre d'art à l'époque de sa reproductibilité technique", Benjamin va jusqu'à écrire la phrase suivante qui choquait profondément Adorno: "Très rétrograde vis-à-vis, par exemple, d'un Picasso, [le public] adopte une attitude progressiste à l'égard, par exemple, d'un Chaplin" (BENJAMIN, 2000a, v. III, p. 100; ADORNO; BENJAMIN, 2002, p. 184-191). Non seulement la révolution des formes par les avantgardes n'est pas, en soi, révolutionnaire, mais elle peut générer une attitude réactionnaire. L'illusion avant-gardiste est, pour un Benjamin ici plus proche de Brecht que d'Adorno, celle de membres d'une classe bourgeoise inapte à rapprocher des masses leur art soi-disant "supérieur": "Quiconque a vu cela, écrit-il, sera enclin à rabattre les prétentions du film abstrait - quelque importantes que soient ses expérimentations." (BENJAMIN, 1989, p. 413). La simple transformation des formes ne suffit pas à assurer à elle seule la fonction révolutionnaire (au sens social et politique) de l'œuvre d'art. Dans son texte d'intervention au congrès antifasciste, Benjamin va plus loin et pointe les pièges mortels: à trop souligner la nécessaire rénovation des formes, les révolutionnaires en art pourraient se retrouver parmi les fascistes à demander une “ rénovation spirituelle " (BENJAMIN, 2003, p. 132); à trop insister sur l'individu (Picasso), ils risquent malgré eux de soutenir les réactionnaires qui en appellent au retour de la "personnalité créatrice" (BENJAMIN, 2003, p. 137).

7 BERL (Emmanuel), "Premier pamphlet". Europe, n. 75, 1929. Dans l'essai qu'il leur consacre, Benjamin (2000a, v. II, p. 131) critique les surréalistes pour être trop "romantiques" et pas assez "dialectiques". Il pourrait enregistrer cette critique comme du même tonneau: les surréalistes seraient naïvement romantiques quant à leur surévaluation de la révolution des formes (Picasso), et ne considèreraient pas assez la dialectique du contenu et de la forme dont le moteur est l'innovation technique au sein des moyens de production. Dans son commentaire de la note de Berl, il note que l'œuvre d'art doit "s'ouvrir dialectiquement au kitsch" (1989, p. 413). Sur la position ambiguë et parfois injuste de Benjamin vis-à-vis du surréalisme, voir Löwy (1996, 2013) et Lachaud (2014, p. 71-93). 
Dès le début de son texte sur la reproductibilité, Benjamin (2000a, v. III, p. 69) met en garde contre la restauration des "concepts traditionnels" comme "création", "génie" ou "valeur d'éternité" trop facilement consonants avec les fascismes de l'époque. Abel Gance, par exemple, réclame pour le film le même respect dû au hiéroglyphe et le même type de "culte" (BENJAMIN, 2000a, v. III, p. 85). Benjamin tire le signal d'alarme: une restauration de la forme créative, géniale ou éternelle reviendrait à une tentative réactionnaire de restauration de l'aura et des dispositifs rituels autour de l'œuvre d'art. ${ }^{8}$ L'œuvre d'art se définissait en effet, avant que sonne l'heure de sa reproductibilité, par son caractère rituel. Son modèle le plus ancien était le sacrifice religieux que l'on faisait au pied du totem (BENJAMIN, 1991, p. 148). Le principe de recueillement devant un poème de Rainer Maria Rilke ou un tableau d'André Derain (BENJAMIN, 2000a, v. III, p. 308) ne forme en ce sens qu'un résidu de cette "valeur rituelle" de l'œuvre d'art. Les regards que l'on portait sur elle (du prêtre, du sacrificateur, puis de l'esthète, du collectionneur, du conservateur, etc.) formaient la couche anthropologique qui la soutenait: ils la gonflaient d'aura, lui prêtaient un pouvoir magique, religieux ou simplement spirituel, celui que Benjamin remarque dans le regard jeté en retour par l'œuvre sur celui qui la contemple:

L'expérience de l'aura repose donc sur le transfert, au niveau des rapports entre l'inanimé - ou la nature - et l'homme, d'une forme de réaction courante dans la société humaine. Dès qu’on est - ou qu'on se croit regardé, on lève les yeux. Sentir l'aura d'une chose, c'est lui conférer le pouvoir de lever les yeux. (BENJAMIN, 2002, p. 200).

L'œuvre d'art était auratique parce que les hommes y avaient transféré un type de relation qu'ils entretenaient avec ceux qu'ils considéraient comme leurs égaux, et qui renvoyait à une certaine réciprocité. L'apparence magique provenait de cette projection anthropomorphique d'une âme dans l'œuvre d'art elle-même. Or cette projection s'accompagne d'une inégalité sociale: en même temps que le prêtre confère une âme au totem, il désigne une vie humaine qu'on pourra lui sacrifier. De manière similaire, chez Benjamin, le respect exagéré que portent les dominants aux œuvres d'art s'accompagne d'un mépris structurel pour les dominés. C'est le sens profond de sa phrase

\footnotetext{
${ }^{8}$ Georges Didi-Huberman ne cesse de prouver, dans ses livres depuis vingt ans, la véracité d'une telle intuition par exemple (DIDI-HUBERMAN, 1992), et tente lui-même, dans ses récentes pratiques d'exposition (et notamment avec le photographe Arno Ginsinger), d'aller à rebours de la valeur rituelle de l'exposition propre à l'industrie mondialisée de la culture et des musées.
} 
souvent citée selon laquelle tout document de culture est en même temps un document de barbarie. Elle signifie: pas d'œuvre d'art sans le sacrifice anonyme de plusieurs générations d'esclaves, de serfs ou de prolétaires (BENJAMIN, 2000a, v. 3, p. 187, 1989, p. 482, 485). L'aura de l'œuvre d'art renvoie ainsi aux illusions qui accompagnent la domination, au point qu'on pourrait la comprendre comme une esthétisation accompagnant l'économie politique. Il n'est donc pas étonnant que les fascistes cherchent à la renforcer dans leur projet d'esthétisation du politique tout court. Lorsque ces illusions, au contraire, se dissolvent, l'oppresseur ne rencontre plus le regard de l'œuvre, mais celui, hostile, de l'opprimé, ressenti "comme gênant, voire nuisible" par l'oppresseur:

Autant d'aura dans le monde qu'il y a de rêve en lui. Mais l'œil éveillé ne perd pas l'art du regard quand le rêve en lui est éteint. Au contraire, ce n'est qu'alors que le regard devient vraiment pénétrant. Il cesse de ressembler au regard de l'amante [ou de l'œuvre] qui sous le regard de l'amant [ou du spectateur] lève les yeux; il commence à ressembler davantage au regard par lequel le méprisé répond au regard du contempteur, l’opprimé au regard de l'oppresseur. De ce regard, tout lointain est effacé; c'est le regard de celui qui s'est éveillé de tout rêve, aussi bien nocturne que diurne." (BENJAMIN, 2013b, p. 29).

Aussi la lutte de classes s'accompagne-t-elle aussi d'une lutte pour contrer le regard de l'ennemi de classe, et une dialectique du regard mise au jour par Benjamin participe-t-elle du combat.' Si Benjamin invalide l'effet politique des révolutions formelles de l'avant-garde, c'est pour libérer le regard de l'opprimé prisonnier du cercle auratique de l'œuvre d'art, c'est-àdire du culte des formes dans lequel se situe encore la volonté de révolutionner les formes. Or le jeu avec la technique (trucages, montages saccadés, images animées) sort plus radicalement du culte que les expérimentations formelles. C'est pourquoi, parmi "les premiers films surréalistes", Benjamin ne nomme pas ceux de Luis Buñuel et Salvador Dali, mais "Méliès, Chaplin, Disney" (BENJAMIN, 1989, p. 97 et 414, 2000a, v. II, p. 371-372) ${ }^{10}$, c'est-àdire des films "grand-public", films à trucages, films burlesques et dessins animés. Si ces films sont, aux yeux du philosophe, plus révolutionnaires que les films expérimentaux, c'est parce qu'ils apportent "un peu de chaleur" (BENJAMIN, 1989, p. 413) au public, et réactivent ce faisant une certaine

\footnotetext{
${ }^{9}$ Pour un commentaire plus approfondi de cette citation, voir BERDET, 2014, p. 213 et suivantes.

${ }^{10}$ Sur Chaplin et Disney chez Benjamin, voir (LESLIE, 2002; LINDNER, 2004 ; HANSEN, 2012).
} 
"haine" (BENJAMIN, 1989, p. 413) vis-à-vis de l'oppresseur, hostilité incandescente que l'on peut interpréter comme celle des "eaux glacées du calcul égoïste” (MARX; ENGELS, 1998, p. 76) qui, selon Karl Marx et Friedrich Engels dans le Manifeste du parti communiste, caractérisent l'ordre politique et social. Ces films, par leur proximité avec le public, libèrent le regard du voile onirique et auratique déposé sur le monde. Être proche du public ne signifie pourtant pas faire en sorte que le public puisse s'identifier à un sujet principal, un sujet " prolétarien ”, qui aurait remplacé l'ancien, "bourgeois". Il ne suffit pas de remplacer le héros bourgeois par un ouvrier à la chaîne ou par une souris qui subit les violences de la mécanisation de la vie moderne. Pour appréhender Chaplin et Disney comme révolutionnaires, il faut encore comprendre la critique de la position qui, aux antipodes des avant-gardes, milite pour la révolution du contenu, et n'aboutit qu'à son remplacement.

\section{REMPLACER LE CONTENU}

La révolution du contenu renvoie trop souvent, d'après Benjamin (2003, p. 130), à une simple substitution aux effets "réactionnaires". Il ne s'agirait que d'utiliser les mêmes moyens en changeant de sujet: mettez un pauvre à la place d'un riche dans le même cadre, et vous aurez le nouveau sujet de l'histoire. Or à en croire Benjamin, si l'artiste se contente d'utiliser les moyens de production tels qu'ils existent, si, photographe, il ne se met pas à écrire une légende qui questionne la prise de vue (BENJAMIN, 2003, p. 134), si, écrivain, il n'introduit pas l'image cinématographique dans l'expérience solitaire du lecteur, si tout cinéaste, photographe ou écrivain ne modifie pas la division du travail au sein du processus de production du film, de la photographie ou du livre lui-même, bref s'il ne fait pas tomber l'une de ces multiples barrières dressées entre les différents producteurs, dont celle entre le manuel et l'intellectuel n'est pas des moindres, alors son travail aura des effets contre-révolutionnaires. Mettons qu'il photographie la misère sans rien changer au reste: alors il n'aura réussi qu'à l'esthétiser, c'est-à-dire, à l'instar du photographe de la Nouvelle objectivité Albert Renger-Patzsch (BENJAMIN, 2003, p. 134), à en faire un objet de jouissance esthétique comme un autre et même, peut-être comme Sebastiáo Salgado aujourd'hui (STALLABRASS, 1997), à "faire de la misère un objet de consommation" (BENJAMIN, 2003, p. 136). 
Une telle pente propre aux artistes de la Nouvelle objectivité et à certains écrivains qui se disent socialistes peut aller jusqu’à "[...] faire du combat contre la misère un objet de consommation" (BENJAMIN, 2003, p. 136). Dans ses ouvrages sur Baudelaire et Paris, le philosophe souligne combien, depuis la fin du XIXe siècle, les écrivains concurrents qui ne peuvent plus compter sur les mécènes se taillent des parts de marché (BENJAMIN, 2002, p. 159; BENJAMIN, 1989, p. 287-288) et s'arrachent - notamment Hugo et Lamartine - la figure du peuple à des fins de populisme littéraire (1989, p. 722, 760, 771, 773, 777). Le gauchisme peut en effet se réduire - et il n'y a pas de raison pour qu'aujourd'hui ce ne soit plus le cas - à une pose intellectuelle, un signe distinctif, tel le romantisme ou le classicisme, sur le marché aux produits culturels, comme ce fut le cas pour des écrivains comme Erich Kästner, Franz Mehring ou Kurt Tucholsky brocardés dans "L'auteur comme producteur" (BENJAMIN, 2003, p. 136). Quand elle n'est pas simplement opportuniste, cette attitude littéraire musèle le peuple au nom duquel elle prétend parler. Et elle lui fait la leçon. Si l'activisme allemand suit aussitôt la Nouvelle objectivité (les photos “objectives" de Renger-Patzsch) parmi les exemples de Benjamin, c'est, semble-t-il, parce qu'il rend explicite la position altière de la "tendance " révolutionnaire qui leur est commune: l'artiste ne veut pas seulement suivre et accompagner la révolution en cours, mais surtout la précéder. Aussi Kurt Hiller et Alfred Döblin, dans leurs œuvres paternalistes et anticommunistes Logocratie (1921) et Connaître et transformer (1931), se posent-ils en intellectuels éclairés au-dessus de la foule pour mieux en diriger les affects (BENJAMIN, 2003, p. 131). Là encore, le travail militant est soumis à l'ordre du sensible, et utilise des moyens conventionnels pour gagner les dominés à leur propre cause que, sans les philosophes aspirant rois (futurs logocrates), ils ignoreraient.

À lire Benjamin, on a l'impression que si cette autoproclamée "tendance révolutionnaire" a, en fin de compte, des effets contre-révolutionnaires voire réactionnaires, c'est parce qu'elle ne fait pas tomber la barrière principale: en refusant de modifier ses propres conditions de production, elle évite de remettre en cause l'ultime barrière, celle entre travail intellectuel et manuel, entre celui qui sait et celui qui fait, entre l'artiste et son public, antagonisme qui se réifie, sur le marché des produits culturels, entre une marchandise animée et un consommateur passif. Ladite "tendance" révolutionnaire semble partir de l'hypothèse d'une inégale intelligence: l'artiste serait bien capable de modifier les formes qu'il utilise (c'est, après tout, son travail), mais l'ouvrier ne le comprendrait pas. Il faudrait donc utiliser des formes éculées pour bien 
se faire comprendre. C'est peut-être pourquoi les productions qui se veulent militantes restent bien souvent sérieuses, grises, et ne s'épanouissent pas dans l'explosion de couleurs de la subversion des formes. Et pourtant, on l'a vu, on ne peut faire jouer cette dernière contre la simple substitution de contenu. Benjamin renvoie dos à dos forme et contenu: comme la révolution des formes, celle du contenu, pour être valable, présuppose chez lui le collectif plutôt que l'individu, la production plutôt que la création et l'organisation du travail plutôt que l'inspiration géniale. Il ne s'agit donc plus de la révolution superficielle du contenu, contrainte au seul sujet de l'art, ni de celle des formes, limitée à la manière de le traiter, mais de quelque chose de beaucoup plus profondément inscrit dans la structure sociale: de la révolution du socle des rapports de production. Il s'agit, au fond, de changer la technique.

\section{Changer la techniQue}

Au-delà du remplacement de contenu et de la rénovation formelle, Benjamin propose de transformer l'œuvre d'art en bouleversant sa technique de production: "Il ne s'agit pas de rénovation spirituelle comme la proclament les fascistes; au contraire on propose des innovations techniques." (BENJAMIN, 2003, p. 132) Cet argument central de "L'auteur comme producteur" se retrouve au cœur de son texte sur la reproductibilité, et permet de mieux comprendre pourquoi Chaplin, Méliès et Disney sont plus révolutionnaires que Picasso, Buñuel et Renger-Patzsch: "La possibilité technique de reproduire l'œuvre d'art modifie l'attitude de la masse à l'égard de l'art. Très rétrograde vis-à-vis, par exemple, d'un Picasso, elle adopte une attitude progressiste à l'égard, par exemple, d'un Chaplin" (BENJAMIN, 2000a, v. III, p. 100). Ce simple passage, qu'un Adorno défenseur de la musique avant-gardiste de Schönberg aurait aimé supprimer du texte (ADORNO; BENJAMIN, 2002, p. 188), résume pourtant la position de Benjamin qui relie la nécessité politique d'innovations techniques au sein de l'œuvre d'art ("L'auteur comme producteur") à la possibilité technique de sa reproduction ("L'œuvre d'art à l'époque de sa reproductibilité technique") et transfère du même coup la technique révolutionnaire du producteur (Chaplin) à son public (la salle de cinéma, pleine d'ouvriers et d'employés).

La reproductibilité de l'œuvre d'art, qui culmine dans le film, a précipité le déclin de son aura, c'est-à-dire l'auréole religieuse ou spirituelle devant laquelle le spectateur devait s'incliner et se recueillir. Elle a, du même 
coup, fait disparaître le brouillard onirique dans lequel se perdait le regard de l'opprimé et qui conférait à l'œuvre, plutôt qu'à l'opprimé, le pouvoir de lever les yeux. Circonscrit dans le domaine des formes esthétiques qu'il révolutionne, Picasso ne peut pas provoquer une attitude révolutionnaire de la part de la majorité, puisqu'il s'inscrit dans la tradition rituelle qui emprisonne le regard dans un voile onirique: de même que l'aura est "[...] l'unique apparition d'un lointain, aussi proche soit-il” (BENJAMIN, 2000a, v. III, p. 75), Picasso nous éloigne d'un monde pourtant proche. Au contraire Chaplin, parce qu'il permet de voir qu'à l'origine de l'œuvre d'art se trouve non pas une force sacrée, mais l'organisation sociale des forces productives, c'est-à-dire une communauté humaine de techniciens et d'ingénieurs et un ordre social et politique qui la soutient, et parce qu'il joue explicitement avec un tel dispositif plutôt qu'il ne nous le dissimule, nous rend le monde industriel plus proche, et nous réchauffe par le rire.

On pense, bien sûr, à l'ouvrier pris comme un grain de sable dans l'engrenage de l'usine des Temps modernes (1936). Mais peut-être qu'un de ses premiers films, muet, montrera mieux encore combien, dès son apparition, Charlot et le spectateur font ensemble l'apprentissage ludique des possibilités de la technique, en l'occurrence de la caméra. ${ }^{11}$ Kid Auto Races at Venice (1914) met en scène une suite de gags à partir du même ressort: Charlot, présent à une course de voiture filmée, veut lui aussi être filmé. La caméra de Chaplin filme ainsi Charlot entrant, par toutes sortes de subterfuges (l'air de rien, en vitesse, en force etc.), dans ce qu'il devine être le champ de la caméra filmant le spectacle. Le spectateur réjoui assiste aux pantomimes de ce clown amoureux de la lentille de la caméra dans laquelle il veut se mirer, et qui se fait chasser sans relâche du champ par l'assistant exaspéré, par l'opérateur lui-même et même par un policier. Tout comme L'homme à la caméra (1929) de Dziga Vertov, ce court-métrage met en scène le dispositif même du cinéma: l'opérateur qui tourne la manivelle, l'assistant qui organise le cadre et même la police qui en chasse l'indésirable. Tout cela est filmé, mise en abîme humoristique adressée au spectateur. L'artiste n'efface pas, comme dans l'art auratique similaire en ce point aux fantasmagories du capital ${ }^{12}$, les traces du processus de production,

\footnotetext{
${ }^{11}$ Pour une autre interprétation, voir l'analyse stimulante de Comolli (2009, p. 42-45).

${ }^{12}$ Ce qu'a d'ailleurs souligné Adorno dans des passages de son Esthétique où il systématise nombre d'intuitions de Benjamin : "Au XIXe siècle, l'apparence esthétique s'était haussée en fantasmagorie. Les œuvres d'arts effaçaient les traces de leur production; probablement parce que l'esprit positiviste croissant de l'art se communiquait dans la mesure où il devait être un fait et avait honte de ce par quoi sa dense immédiateté s'était révélée médiatisée." (ADORNO, 2011, p. 140). Il faudrait étudier
} 
mais au contraire les rend visibles. Il met en scène non seulement le droit autoproclamé à être filmé, mais aussi les conditions de production et, pourquoi pas, la possibilité de passer derrière la caméra à la place de l'opérateur pour faire d'autres choix (par exemple: filmer le jeu heurté d'un vagabond plutôt que le circuit fluide d'une course de voitures).

Aussi anecdotique qu'il puisse paraître, ce film, qui contient en puissance le jeu de Chaplin avec l'appareil (les saccades de l'acteur qui répètent celles du montage, les clins d'œil au spectateur, ce coup de chapeau insolent complice d'une éruption joyeuse qui dérègle l'ordre bourgeois, etc.), fait quelque chose que ne peut pas faire Picasso, et qui est de première importance pour Benjamin: il familiarise l'humanité avec ses nouveaux appareils. Le cinéma, lorsqu'il ne cherche pas, avec Abel Gance, à reproduire l'aura traditionnelle de l'œuvre d'art, permet à l'homme d'apprivoiser le nouvel appareillage technique créé par la révolution industrielle: il ouvre un nouvel espace de jeu, un nouveau Spielraum (WOHLFARTH, 2014). Autrement dit, il prépare l'homme à une mise en commun des forces techniques, et cela afin de réaliser les buts utopiques de l'humanité. Benjamin remarque en effet deux fonctions de l'art que le film a permis d'arracher à l'emprise rituelle sur l'œuvre:

1) familiariser l'humanité avec des images déterminées, avant même que les finalités poursuivies par ces images soient devenues conscientes; 2) permettre aux tendances sociales dont la réalisation [Realisierung] aurait un effet destructeur sur les hommes eux-mêmes de conquérir leur droit dans le monde des images. (BENJAMIN, 1991, p. 181).

Une fonction utopique, une fonction cathartique. Les moments poétiques de Chaplin, les séquences oniriques de Méliès ou, plus encore, l'animation fantastique de Disney remplissent la première fonction. C'est Mickey Mouse qui, pour Benjamin (2000a, v. II, p. 371-372), incarne au mieux l'image utopique d'une existence libérée où la nature et la technique, les nuages et les machines concourent ensemble à la symphonie d'un monde merveilleux. Dans ses films d'avant 1939, Disney met en scène l'innervation technique du collectif (BENJAMIN, 1991, p. 149) rêvée, un siècle plus tôt, par Charles Fourier dans ses fantastiques utopies nées de la contemplation des

la circulation du concept de fantasmagorie entre Adorno et Benjamin (et peut-être aussi Siegfried Kracauer) et son utilité pour une esthétique post-auratique à l'heure de la marchandisation de l'œuvre d'art. Il s'agit là d'un terrain de recherche fécond, pour l'instant inexploré, qui permettrait de penser l'esthétique du capitalisme jusqu'à aujourd'hui. Pour des premiers pas dans cette direction, je me permets de renvoyer à mon propre travail, en particulier Berdet $(2013,2015)$. 
nouvelles possibilités de la technique industrielle (BENJAMIN, 1989, p. 3637; aussi BERDET, 2013, p. 37-72, 155-164). Quant à la fonction cathartique, elle se manifeste dans le rire des spectateurs généré par la maltraitance du vagabond au chapeau melon ou de la petite souris noire: pour Benjamin, tant qu'elle peut s'actualiser par le rire dans la salle de cinéma, la violence sadique du collectif ne se manifestera pas dans l'histoire. Il semble même que les deux fonctions soient liées, et que le public qui a pu sublimer ses tendances sadiques devant un slapstick pourra d'autant plus facilement réaliser pacifiquement les images utopiques dont rêve l'humanité depuis longtemps.

Il existe pour Benjamin une correspondance entre l'innovation technique de l'artiste (le montage saccadé de Chaplin, les trucages de Méliès, la plasticité du dessin filmé de Disney) et la capacité collective à se saisir des nouvelles possibilités techniques: de même que l'artiste sera capable de révolutionner son mode de production, la "masse", c'est-à-dire la majorité du public, c'est-à-dire les ouvriers et les employés, seront capables de révolutionner les leurs (BENJAMIN. 2003, p. 138). Cette révolution passe non pas par un individu isolé, mais par un collectif organisé, et c'est pourquoi Benjamin préfere mobiliser le film, avec son équipe de techniciens, qu'un autre type d'œuvre d'art: au cours de la production d'un film, tout le monde a, potentiellement, son mot à dire, et l'acteur, dont la présence auratique a été d'abord décomposée par la suite de plans ${ }^{13}$, ne devrait pas être plus important que l'éclairagiste ou le preneur de son (BENJAMIN, 2000a, v. III, p. 94-98). De plus, le cinéma est par excellence l'art qui déborde sur les autres arts (théâtre, photographie, musique...) pour remettre en question les cloisons étanches entre les disciplines, non pas dans l'optique d'une "œuvre d'art totale" (BENJAMIN, 2003, p. 141), mais plutôt, dans la lignée du "laboratoire théâtral " de Brecht (ibid.), dans celle d'un laboratoire filmique. Il va jusqu'à refaire le partage entre l'artiste et son public, les individus filmés pouvant se faire filmant.

Le film, art reproductible, perfectible et collectif par excellence, permet à Benjamin - qui écrit dès 1927 sur le film soviétique et l'art collectiviste ${ }^{14}$ - d'illustrer au mieux son idéal d'intervention technique dans les moyens de

\footnotetext{
${ }^{13}$ Elle sera recomposée par son aura de "star" produite par l'industrie du divertissement, comme Benjamin en a l'intuition : (BENJAMIN, 2000a, v. III, p. 93-94).

14 "Sur la situation du film russe" et "Réponse à Oscar A. H. Schmitz", 1972-1999, v. II, p. 747-755. Ces deux textes ont été traduits par les Cahiers du cinéma de janvier-février 1971 consacrés à Serguei Eisenstein. Benjamin écrit aussi sur la situation des écrivains russes, du poème russe ou encore sur le programme d'un théâtre prolétarien pour enfants. Sur Benjamin, Brecht et l'art communiste, voir les
} 
production esthétique. Dans "Lauteur comme producteur", le philosophe proposait d'autres exemples, comme le russe Sergueï Tretiakov qui, proche de Bertolt Brecht, Vladimir Maïakovski, Vsevolod Meyerhold et Sergueï Eisenstein, avait délaissé sa plume d'écrivain privé pour militer dans les kolkhozes tout en en racontant l'histoire, ou encore la presse soviétique qui permettait aux lecteurs de devenir rédacteurs (BENJAMIN, 2003, p. 125-129). De tels exemples, peuvent sembler moins convaincants (on a l'impression que la double casquette de Tretiakov fait plutôt de lui un intellectuel organique au sens classique du terme décrit par Antonio Gramsci, et l'optimisme de Benjamin quant à la capacité de la presse soviétique - organique elle aussi - à ouvrir ses colonnes à son public paraît rétrospectivement bien naïf). Ils montrent cependant combien il s'agit avant tout, par l'élargissement d'une compétence technique, de bousculer la division bourgeoise du travail. A l'heure où chacun peut publier un texte, une image ou une vidéo sur internet, et malgré leurs limites (et peut-être en vertu même de ces limites), ils seraient peut-être à reconsidérer. Ce n'est pas le lieu d'en discuter ici, mais en tout cas, l'idée d'intervenir, par la technique, en "ingénieur" et en "spécialiste" (BENJAMIN, 2003, p. 143) plutôt qu'en théoricien et en généraliste, dépasse les présupposés de l'idéologie de la communication, d'après laquelle il suffirait de changer le message (le contenu) ou le médium (la forme) pour avoir un quelconque effet sur le lecteur, le spectateur ou l'auditeur.

\section{Conclusion: Au-delà de la COMmunication}

L'anecdote du café Tiergarten rapportée par Scholem recèle, en germe, non seulement l'esthétique de Benjamin, mais aussi sa politique de l'esthétique, c'est-à-dire sa manière d'intervenir dans l'ordre esthétique même. Elle tient en une phrase, mais dont les conséquences sont redoutables et mettent à mal bien des clichés: il ne s'agit pas de transmettre un message, mais de transformer le langage. Ce défi ne s'adresse pas seulement aux artistes, écrivains et cinéastes qui, encore aujourd'hui, souhaitent œuvrer à l'émancipation. Il vise aussi, à travers eux, les idéologues de la communication. Enseignants ou étudiants, politiciens ou conseillers du prince, publicitaires ou, précisément, "communicants", nombreux sont ceux qui voudraient pouvoir utiliser (ou, pour faire chic, citer) le penseur de la reproductibilité technique, des nouveaux médias et du langage humain pour leurs propres fins.

nombreux textes de Philippe Ivernel, qu'hélas aucun éditeur n'a encore rassemblés, et en particulier Ivernel $(1987,1988,1989)$. 
Pourtant, près de 80 ans avant la vogue de la communication tous azimuts, le philosophe allemand en avait sapé la base, selon laquelle tout se passerait entre un individu émetteur et un individu récepteur, comme si le langage propre à l'homme se limitait à un signal électrique circulant entre deux antennes. Même si l'on étend aujourd'hui le message au média, la conception reste la même: une personne "communique" un message à une autre par le biais d'un instrument, que ce signifiant soit réduit à sa plus simple expression (le mot qui signifie la chose) ou pris dans un maillage technique qui peut à l'occasion surdéterminer son sens (l'image, le son, le rythme, le montage, le cadrage...), bref à son "contenu" ou à sa "forme". C'est bien souvent, hélas, le point de vue qui soutient encore des films militants pleins de bonnes intentions. Ceux-ci croient pouvoir émanciper en mobilisant les mots, les formes et les dispositifs techniques de l'adversaire pour les retourner contre lui: il suffirait d'utiliser les mêmes moyens pour d'autres fins. Or dès 1916, le verdict de Benjamin était implacable: "Cette vue est la conception bourgeoise du langage, dont la suite [du texte: "Sur le langage en général et le langage humain"] va montrer de plus en plus clairement le caractère intenable et vide. Cette vue consiste à dire: le moyen de la communication est le mot, son objet est la chose, son destinataire est l'homme." (BENJAMIN, 2000a, v. I, p. 147).

On utilise donc (a) le mot comme instrument pour communiquer (b) la chose à destination de (c) l'homme. C'est l'abc de la communication: trois points, trois lignes, un triangle parfait, lisse, sans bavure, sans aspérité et surtout sans perte. Benjamin lui oppose une fonction expressive du langage: le mot (mais cela sera valable aussi bien pour l'image et le montage) exprime toujours plus que ce qu'on veut communiquer, il est dépositaire d'un surplus de sens. En tout cas en puissance, c'est-à-dire lorsque le potentiel du langage n'est pas écrasé par les conventions. Dans "Sur le langage en général et le langage humain” (1916), le récit de la Bible permet à Benjamin de générer un écart par rapport à l'usage commun du langage, un peu comme le récit du bon sauvage permet à Rousseau de s'écarter de la civilisation pour mieux la critiquer: le langage " pur" du paradis adamique (où le mot et la chose coïncident directement, où le monde réel est fait de langage, et le langage de monde réel) permet de voir, même si c'est une fiction, combien le langage profane des modernes est étouffé par la raison instrumentale. La puissance du langage peut alors se mesurer à sa capacité à créer un surplus d'existence plutôt que de s'épuiser à reproduire les conventions. D'où la thèse centrale de Benjamin: dans le langage vivant, le mot est en puissance porteur d'un excès de sens, d'un réel en surplus qui ne s'épuise pas dans la communication. 
Lorsque le poète trouve le mot juste, qu'il organise sa syntaxe d'une certaine manière, alors il ne se contente pas de délivrer un message selon l'agencement connu des mots et des choses, mais crée une résonance nouvelle, il fait sortir de l'ombre quelque sensation ou sentiment qui n'existait pas jusque-là et qui oblige à un nouveau partage du monde, un être nouveau s'y étant fait une place.

Le cas du poète est un cas-limite vers lequel tend Benjamin dans son texte. Mais cela ne l'empêche pas de considérer que les langages de la musique, de la sculpture, de la justice et de la technique (BENJAMIN, 2000a, v. I, p. 142) peuvent être eux aussi chargés d'un surplus de spiritualité: un son inédit, une forme jamais vue, un droit nouveau ou une technologie qui libère les potentialités de l'homme et de la nature sont en effet des éléments de langage qui élargissent le monde spirituel de l'homme. A contrario, on pourrait nommer des formes de langage appauvrissantes: un spot publicitaire débilitant, une statue monumentale comme le pouvoir qui l'érige, une nouvelle frontière qui, par essence, enlève des droits à tous pour donner une propriété à quelques-uns ou encore une technologie qui oppresse la nature et inquiète les hommes (le nucléaire ?). Un langage qui, pris dans le triangle idéologique de la communication, se voit comme un moyen en vue d'une fin, est soumis à une raison instrumentale liée à la domination de l'homme sur l'homme et de l'homme sur la nature. Dans sa dimension expressive, le langage sort de cette logique moyen-fin, mot-lecteur, message-spectateur qui abrutit le destinataire en le tenant, précisément, pour incapable d'accueillir une impression nouvelle: il génère un nouveau site que nous ne pouvons appréhender avec les mots ou les images de tous les jours, il nous oblige au fond à vivre avec cette étrangeté, à refondre notre grammaire intime pour y reconnaître quelque chose de nouveau. ${ }^{15}$

Il ne s'agit pas là d'une position hostile à la technique qui s'en prendrait aux nouveaux moyens de communication au nom d'un bon vieux sens perdu. Walter Benjamin s'enthousiasme pour les possibilités offertes par la photographie, la radio et le cinéma. Cependant, il ne faudrait pas prendre non plus un texte comme "L'œuvre d'art à l'époque de sa reproductibilité technique" pour un retournement qui contredirait sa position de 1916. Il y a certes, de 1916 à 1935, rupture (de l'idéalisme au matérialisme), mais aussi

\footnotetext{
${ }^{15}$ Georges Didi-Huberman, dont il faudrait lire chaque ouvrage comme une poursuite de l'esthétique benjaminienne, a souligné cela à partir de "Petite histoire de la photographie": (DIDI-HUBERMAN, 2012, p. 17).
} 
continuité (la puissance expressive du langage déposée dans les pouvoirs de la technique moderne), changement d'objet (du paradis linguistique d'Adam à l'usine filmique de Chaplin) mais pas tout à fait d'optique. Même s'il flirte avec chacune de ces positions, on ne peut réduire Benjamin ni à son objet de 1916, la Bible, ce qui ferait de lui un rabbin talmudique; ni à son objet de 1935, le cinéma, ce qui ferait de lui un technophile soviétique. Le philosophe allemand n'est pas à ranger, comme le voudrait Jacques Rancière (2000, p. 47), aux côtés de Martin Heidegger parmi les nostalgiques d'une ontologie perdue, d'une origine évanouie (grecque ou biblique). Mais on ne peut pas le classer non plus parmi les enthousiastes naifs de la technique que soupçonnait en lui Adorno, comme s'il s'enfermait lui-même dans les fantasmagories capitalistes qu'il dénonçait (ADORNO; BENJAMIN, 2002, p. 117, 133, 135, 139, 187-189, 361 par exemple $\left.^{16}\right)$. Entre sa critique de la communication au nom de la puissance expressive du langage (1916) et sa critique de l'esthétique militante au nom de l'intervention dans l'appareil de production (1934), il existe une profonde continuité, au point qu'on peut comprendre la seconde comme la reprise (certes matérialiste) de la première. Dans son essai sur la reproductibilité de 1935, Benjamin introduit cette critique dans l'appareil technique lui-même (le cinématographe), porteur, tout comme le simple mot, d'un surplus de sens irréductible à sa fonction instrumentale: le film n'est pas le simple véhicule d'un message, il en dit toujours plus, ou plus exactement il intervient à plusieurs niveaux du partage du sensible, de sa confection à sa diffusion. Allié au Benjamin mystique des années 1910, le Benjamin marxiste des années 1930 ne cesse de critiquer l'utilisation bourgeoise du langage, de la technique et de l'esthétique ${ }^{17}$ afin de rouvrir l'horizon utopique d'un homme réconcilié avec son propre langage et avec la nature, là où, comme il l'écrit dans une note de 1917-1919, “[...] les problèmes moraux s'effacent complètement pour que l'image utopique d'un monde astral spirituel puisse déployer les formes pures et sans ambiguïté de la technique." (BENJAMIN, 1972-1999, v. II, p. 619) ${ }^{18}$.

BERDET, Marc. "Lead to what is denied the word»: Walter Benjamin and the critique of the ideology of communication. Tans/form/ação, Marília, v. 40, n. 3, p. 45-66, Jul./Set., 2017.

\footnotetext{
${ }^{16}$ Sur ces polémiques épistolaires avec Adorno, voir Berdet (2015, p. 58-80).

${ }^{17}$ Avec sa préface épineuse, sa thèse de 1925 voulait déjà bouleverser "le commerce scientifique idéaliste-bourgeois" (BENJAMIN, 1989, p. 43).

${ }^{18}$ J'ai commenté cette note inédite en français dans Berdet (2014, p. 133, 281).
} 


\begin{abstract}
Walter Benjamin is ever more present in today's departments of communication studies, and sometimes even used in the communicants' discourses themselves. But there is something in him which resists this instrumentalization. Indeed, in his work, language (political as well as aesthetical) does not offer itself to the communication of a predefined sense, but instead expresses what remains inaccessible to the word. The German philosopher succeed in liquidating the revolutionary posture of the artist still caught in the ideology of communication. This is neither a matter of substituting the content, nor of renovating the form, but - an apparently modest but radically more ambitious objective - of changing the technique.
\end{abstract}

Keywords: Walter Benjamin. Communication. Art. Language. Revolution.

\title{
RÉFÉRENCES ${ }^{19}$
}

ADORNO, T. Théorie esthétique. Paris: Klincksieck, 2011.

. ; BENJAMIN, W. Correspondance 1928-1940. Paris: La Fabrique, 2002.

ARENDT, H. Vies politiques. Paris: Gallimard, 1974. p. 244-306.

BENJAMIN, W. Gesammelte Schriften. Francfort: Suhrkamp, 1972-1999.

. Correspondance I. Paris: Aubier-Montaigne, 1979.

. Correspondance II. Paris: Aubier-Montaigne, 1980.

. Paris, capitale du xix siècle : le livre des passages. Paris: Editions du Cerf, 1989.

. Ecrits français. Paris: Gallimard, 1991.

. Gesammelte Briefe. Francfort: Suhrkamp, 1995-2000.

. Euvres I-III. Paris: Gallimard, 2000a.

. Origine du drame baroque allemand. Paris: Flammarion, $2000 \mathrm{~b}$.

. Charles Baudelaire. Paris: Payot, 2002.

. Essais sur Brecht. Paris: La Fabrique, 2003.

. Das Kunstwerk im zeitalter seiner technischen reproduzierbarkeit. In: Werke und Nachlass. Kritische Gesamtausgabe v. 16. Berlin: Suhrkamp, 2013a.

. Charles Baudelaire. Paris: La Fabrique, 2013 b.

Sens unique. Paris: Payot, 2013c.

BERDET, M. Fantasmagories du capital : l'invention de la ville-marchandise. Paris: Zones/ La Découverte, 2013.

. Walter Benjamin : la passion dialectique. Paris: Armand Colin, 2014.

${ }_{19}$ Pour une bibliographie plus exhaustive, cf. Lindner (Dir.) (2007, en particulier p. 249-251), et Berdet (2014, p. 289-303, en particulier p. 300-301). 
Le chiffonnier de Paris: Walter Benjamin et les fantasmagories. Paris: Vrin, 2015.

BERMAN, A. L'âge de la traduction. La tâche du traducteur de Walter Benjamin: un commentaire. Paris: Presses Universitaires de Vincennes, 2008.

COMOLLI, J-L. Cinéma contre spectacle. Paris: Verdier, 2009.

DIDI-HUBERMAN, G. Ce que nous voyons, ce qui nous regarde. Paris: Minuit, 1992.

. Peuples exposés, peuples figurants: l'œil de l'histoire, v. 4. Paris: Minuit, 2012.

EILAND, H.; JENNINGS, M. Walter Benjamin: a critical life. Harvard: Harvard University Press, 2014.

HANSEN, M. Cinema and experience: Siegfried Kracauer, Walter Benjamin and Theodor Adorno. Berkeley: University of California Press, 2012. p. 75-206.

IVERNEL, P. Passages de frontières : circulations de l'image épique et dialectique chez Brecht et Benjamin. In: Hors cadre, $n^{\circ}$ 6. Saint-Denis: Presses Universitaires de Vincennes, 1987. p. 133-163.

Benjamin et Brecht, ou le tournant politique de l'esthétique. In: RAULET, G.; FÜRNKAS, J. (Dir.). Weimar: le tournant esthétique. Paris: Anthropos, 1988. p. 45-68.

. Introduction. In: LACIS, A. Profession: révolutionnaire: sur le théâtre prolétarien. Grenoble: PUG, 1989. p. 7-27.

LACHAUD, J.-M. Walter Benjamin: esthétique et politique de l'émancipation. Paris, L'Harmattan, 2014.

LESLIE, E. Mickey Mouse, utopia and Walter Benjamin, In: Hollywood flatlands: animation, critical theory and the avant-garde. Londres; New York: Verso, 2002. p. 81-122.

LINDNER, B. Mickey Mouse und Charlie Chaplin. Benjamins Utopie der Massenkunst . In: SCHÖTTKER, D. (Dir.). Schrift, Bilder, Denken:Walter Benjamin und die Künste. Francfort: Suhrkamp, 2004. p. 144-155.

2007.

. Benjamin Handbuch. Leben-Werk-Wirkung. Stuttgart ; Weimar: JB. Metzler,

LÖWY, M. Walter Benjamin et le surréalisme: histoire d'un enchantement révolutionnaire. Europe, Paris, n. 804, p. 79-90, 1996.

Walter Benjamin et le surréalisme. Anthropology \& Materialism, Paris : Cambridge : Berlin, n. 1, 2013.

MARX, K.; ENGELS, F. Manifeste du parti communiste. Paris: Flammarion, 1998.

RANCIÈRE, J. Le partage du sensible: esthétique et politique. Paris: La Fabrique, 2000.

SCHOLEM, G. Walter Benjamin : histoire d'une amitié. Paris: Calmann Lévy, 1981.

.; BENJAMIN, W. Théologie et utopie: correspondance 1933-1940. Paris: L'Éclat, 2011. 
BERDET, M.

STALLABRASS, J. Sebastiao Salgado and fine art journalism. New Left Review, New York, v. 1, n. 223, p. 131-160, mai-juin 1997.

TACKELS, B. Histoire d'aura: l'œuvre d'art à l'époque de Walter Benjamin. Paris: L'Harmattan, 2001.

WOHLFARTH, I. Spielraum: jeu et enjeu de la seconde techniquechez Walter Benjamin. In: BERDET, M.; EBKE, T. (Dir.). Matérialisme anthropologique et matérialisme de la rencontre: arpenter notre présent avec Walter Benjamin et Louis Althusser. Berlin: Xenomoi, 2014. p. 75-142.

Recebido em 19/01/2016

Aceito em 24/08/2016 